\title{
Oficio, oficiantes y extensión universitaria.Notas a favor de una sociología modesta en una universidad de poros abiertos
}

\author{
Jerónimo Pinedo \\ Secretario de Extensión Facultad de Humanidades y Ciencias de la Educación \\ Universidad Nacional de La Plata, Argentina
}

"Que cada persona sea su propio metodólogo: que cada persona sea su propio teórico:
que la teoría y el método vuelvan a formar parte de la práctica del oficio..."

Wright Mills

En estas notas me propongo desarrollar algunos aspectos del oficio de sociólogos/as en el marco de la extensión universitaria en dos movimientos: el primero consiste en situar la práctica de dicha actividad en la Facultad de Humanidades y Ciencias de la Educación (FaHCE) en el contexto más amplio de la Universidad Nacional de la Plata (UNLP); el segundo, trata de re-colocar el despliegue del oficio en el marco de proyectos de extensión de la FaHCE, prestando especial atención a los modos de involucramiento y los bienes cognitivos que producen los/as sociólogos/as al participar de este tipo de procesos de creación de conocimiento sobre lo social.

Estos dos movimientos que voy a realizar se apoyan sobre ciertos puntos de partida. El primero es que la extensión universitaria forma parte del proceso de producción del conocimiento en las universidades públicas nacionales de la Argentina, más allá de las múltiples dificultades que encuentra esta función específica en sus posibilidades (y probabilidades) de "institucionalización exitosa" (Pérez Rasseti, 2015). Esa producción debería mirarse como la producción de cualquier otro ámbito: un proceso global que se encadena en diferentes etapas, mecanismos, medios, actores, relaciones, insumos y productos, a la vez que exige un examen 
detallado de cómo están constituidos los eslabones de esa cadena. Es tan necesario estudiar las articulaciones entre docencia, investigación y extensión, como tener una descripción clara sobre cómo funciona el eslabón de la extensión universitaria en particular, que sea capaz de superar las retóricas de celebración y pueda afrontar las duras pruebas de realidad (Pinedo, 2019). El segundo punto, enfatiza en la profundización de los desarrollos teóricos y metodológicos que permitan empalmar las relaciones entre las disciplinas universitarias y la extensión. El tercer punto, más que un punto de partida, expresa una hipótesis: lo que solicito en el segundo punto ya está ocurriendo, pero no lo vemos porque buscamos en el nivel equivocado. Me explico, nada de esto que está sucediendo (ahora mismo) puede verse con claridad si sólo nos fijamos en el nivel de las normativas, las políticas, los planes, los proyectos y las declaraciones bien intencionadas de los funcionarios universitarios. La universidad no es únicamente un campo o un sistema cuyo cerebro y/o lógica se organiza a partir de un centro, también se la puede concebir en un sentido reticular como una red que conecta con (o se desconecta de) otras redes sociales. Esta mirada sobre la universidad como un espacio de redes resulta muy pertinente para examinar las actividades de extensión. Como lo ha mostrado Soledad Balerdi en su tesis doctoral (2018), hay que mirar hacia la trama de prácticas, técnicas, categorías, conceptos, teorías y metodologías que una intrincada red de actores movilizan en las fronteras porosas entre la universidad y el territorio para generar sus propios efectos de realidad. A esta innovadora mirada propuesta por Balerdi quiero agregarle en estas notas una conceptualización más. Lo que ella observa muy pertinentemente como modos específicos de involucramiento colectivo que definen el carácter y la especificidad de la acción social de ciertos grupos universitarios (Thévenot, 2016) también pueden ser analizados como modelos de inteligencia o cognición distribuida (Hutchins, 1991) de un tipo particular, donde el conocimiento no está ni en la mente, ni en los planes, ni en los papers de nadie, sino en las relaciones entre las personas y entre las personas y los procesos que traspasan de un lado a otro las membranas que vinculan a la sociedad con la universidad. Mirar estos oficios y lo que hacen sus oficiantes nos puede permitir recoger aprendizajes significativos para las disciplinas universitarias, para comprender las vinculaciones universidad-sociedad realmente existentes y abrir nuestros modos de entender cómo se produce, circula y utiliza el conocimiento en ciencias sociales. Esta diversidad de formas creativas de producir conocimiento desde la universidad pública podría potenciarse si, finalmente, los actores que toman las decisiones en el sistema logran reconocer que, quizá, esta sea una de sus singularidades frente a otros dispositivos institucionales y empresariales que disputan con las propias universidades públicas la calidad y la legitimidad de los bienes cognitivos que producen. ${ }^{1}$

\section{UN CONCEPTO CONTROVERSIAL}

Para no aburrir, pero con la debida obligación de informar al lector/a de qué estamos hablando, en este apartado voy a hacer una serie de breves consideraciones sobre la historia y el presente de la extensión universitaria en nuestra Universidad. Aprovecho para advertir a los lectores que este no es el lugar adecuado para buscar una explicación y un análisis detallado del complejo mundo de la extensión universitaria. Aquí simplemente nos vamos a remitir a poner en contexto la pequea parte de un proceso mucho más amplio que por el momento está fuera de nuestro campo de explicación.

Como la propia palabra lo denota, la extensión nació ilustrada, positivista y difusionista. Una carta del 22 de Marzo de 1917 enviada al Ministerio de Educación de la Nación por Joaquín V. González da cuenta de los sentidos que dejaron la huella de sus comienzos.

En los últimos años (1914-1915) particularmente, se ha notado por esta presidencia, con gran satisfacción, un hecho que denota hasta qué punto ha entrado en el espíritu de nuestra colectividad universitaria, el deseo de extender la enseñanza de sus aulas a todos los que la necesiten y colaborar con la obra en la que la universidad se encuentra empeñada.

Me refiero a la iniciativa nacida y puesta en práctica entre los alumnos del colegio nacional de la universidad, de dictar cursos nocturnos para gente del pueblo en las aulas del establecimiento y a cargo de los estudiantes de los cursos superiores del colegio o de cursos universitarios. 
Es realmente edificante este esfuerzo de los alumnos, quienes guiados por el sólo deseo de combatir la ignorancia y dar, a los que no tienen, un poco de luz espiritual, dedican parte del tiempo que les queda para atender sus tareas de estudiantes y en el momento que más lo necesitan, varias horas por noche para transmitir lo que han aprendido a los que desean aprender. (Castiñeira, 1940, p. 36).

Para González no había duda, el conocimiento se producía en la universidad para luego extenderlo a la sociedad a partir de la difusión cultural, la divulgación científica y la transferencia tecnológica. La extensión ocupaba la función de un engranaje del circuito de la ciencia donde la universidad es la que determinaba el sentido, los problemas y los saberes que debían recibir los actores sociales para lograr cambios en su conducta, hábitos y formas sociales arraigadas en una práctica basada en el concepto de "extender la enseñanza". Situada en el campo de la filantropía, la misión social de la universidad se transformaba en un vector moralizante de los supuestos privilegios a los que podían acceder los estudiantes universitarios. Una retribución en especie frente a una deuda moral contraída. De allí que González destacara el "esfuerzo edificante”, las horas quitadas al estudio y al sueño para combatir la ignorancia y dar luz al pueblo. Este modo de pensar el vínculo universidadsociedad sigue estando presente en nuestras prácticas y nuestros modos de concretar el circuito cienciainnovación-aplicación en los procesos unidireccionales de transferencias de conocimientos y tecnologías en relación a múltiples tipos de actividad productiva, social, sanitaria, educativa, etc. Y si por el momento, esos modos de proceder no son por sí mismos objetables, ya que pueden ser muy útiles y necesarios para abordar diversos problemas sociales, lo que no tenemos que desconocer es que producen un modo de vinculación entre los actores universitarios y los actores sociales con sus características específicas que requiere mucho más análisis acerca de sus dinámicas y resultados.

A la persistencia del paradigma difusionista se le sumaron otros modos de llevar adelante la extensión universitaria. Como sostienen Tomassino y Cano (2016), nuestras universidades han sido históricamente un campo donde pugnan diferentes proyectos políticos académicos capaces de disputar los modos de producir las instituciones universitarias, intentando vincular de manera específica los procesos de enseñanza y creación de conocimiento con las problemáticas de las sociedades nacionales y las necesidades de los sectores populares. Estas disputas han llevado a convertir a la extensión en un concepto controversial que se ha pretendido sustituir por otras denominaciones: vinculación, articulación, responsabilidad social universitaria, compromiso social de la universidad, acción social universitaria, cooperación. Sin embargo, en muchas ocasiones la propensión académica a enfrascarse en largas luchas nominalistas provocó una variación de los nombres que encubría la continuidad de las mismas prácticas.

Un nivel mucho más interesante de este proceso controversial, más allá del universo vocabular proliferante de los universitarios, ha sido el intento de innovación y transformación de las prácticas extensionistas cuyos resultados empíricos requieren de un análisis crítico. Evitando cualquier caricaturización de los pensamientos de Joaquín V. González, lo cierto es que en los últimos veinte años asistimos a una creciente heterogeneidad de las actividades de vinculación entre la universidad y la sociedad que coexisten bajo la misma denominación, pero que representan formas muy distintas de concebir y organizar esos procesos. La heterogeneización y disputa no comenzó hace poco tiempo; desde la década del sesenta, el ingreso paulatino, conflictivo y muchas veces interrumpido, de ciertas tradiciones del pensamiento social latinoamericano como la Educación Popular, que reconoce su raíz en Paulo Freire, las metodologías de Investigación Acción Participativa, cuya referencia primaria son los trabajos de Fals Borda, y los enfoques de Ciencia, Tecnología y Sociedad estimulados por científicos como Jorge Sábato, han tenido un valor cuestionador e innovador de los hábitos universitarios, al problematizar los vínculos establecidos con la sociedad e impulsar la incorporación sistemática de agendas, prioridades y saberes de las sociedades autóctonas, los movimientos sociales y los sectores populares en el mundo universitario. No sólo con el objeto de transformar el vínculo universidadsociedad, sino también con la propuesta de cambiar formas de enseñar e investigar en la universidad. Pero para el caso específico de la UNLP, más allá de reconocer en las décadas del sesenta y setenta intentos de transformación, fue a partir del ciclo político inaugurado por la rebelión popular del 2001 que estos 
procesos comenzaron a sedimentar. Una nueva generación de estudiantes y docentes buscó combinar sus perfiles universitarios con tareas y actividades militantes que implicaban diversos tipos de colaboración con las organizaciones sociales surgidas al calor de la movilización popular. Maristella Svampa llamó a este período el "tiempo extraordinario" en el que fueron haciendo su aparición un conjunto variado de colectivos activistas que no buscaron un encuadramiento partidario, sino la construcción de vínculos territorializados y la recuperación y el intercambio de saberes con las organizaciones populares desde los espacios y conocimientos específicos (profesionales, científicos o artísticos) donde se insertaban, configurando así una nueva cartografía de las resistencias, los movimientos sociales y los compromisos y solidaridades de clase. Los derechos humanos, la horizontalidad, la protesta social, la articulación y el diálogo de saberes, pasaron a ser parte de una narrativa militante común entre experiencias diversas y se vio reflejada en las actividades de extensión que surgieron en la UNLP después del 2001.

El programa de extensión universitaria "El derecho a tener derechos" se basa en la formación y capacitación en derechos humanos - derecho a la protesta social, derecho a la ciudad, derechos frente a la violencia policial, derecho a la salud- y está destinado a organizaciones y movimientos sociales (...) se pretende atender problemáticas vinculadas al ejercicio de los derechos, con el fin de fortalecer las capacidades políticas y estratégicas de las organizaciones. El telón de fondo de esta experiencia consiste en la articulación de la Universidad con los sectores populares, generando espacios de diálogo y aprendizaje colectivos y conjuntos. (El derecho a tener derechos, 2006).

La ejecución de Programas de Extensión Universitaria como "El derecho a tener derechos" o "Educación y acceso a derechos en los barrios La Unión y El Mercadito", así como otras múltiples iniciativas que por razones de espacio no podemos citar acá, fueron preparando el terreno para que, hacia finales de la década del 2000, durante el período de reforma del estatuto de la Universidad, madurara una voluntad reformista que colocó a la extensión universitaria en el centro de sus preocupaciones. Estos cambios y alternativas se vieron reflejados no sólo en la separación conceptual y normativa entre extensión y transferencia, sino también en la implementación de políticas que crearon nuevos espacios de interacción entre la universidad y los territorios, como son los Centros Comunitarios de Extensión Universitaria, y entre la universidad y los movimientos y organizaciones sociales como lo es el Consejo Social de la UNLP. Desde ese momento, y en torno a estas nuevas orientaciones de la política de extensión, fueron surgiendo numerosas alternativas. Sin embargo, no hay que confundir la efectiva recreación de las condiciones político-institucionales con la construcción de un diálogo de saberes y una ecología de los conocimientos. Si necesarias, esas condiciones no son suficientes, en la medida que el alcance de esas metas está mediado por la posibilidad de producir los insumos teóricos y metodológicos que no están del todo disponibles y que es necesario imaginar e inventar, empresa creativa en la que el ejercicio del oficio sociológico hace su contribución.

Volviendo a la hipótesis que enunciamos al principio de estas notas, quiero sostener que las fuentes de esa imaginación no está en los diseños institucionales y ni en las estructuras políticas, administrativas y presupuestarias del sistema universitario, sino en la experiencia de los equipos extensionistas largamente entrenados en la realización de actividades en espacios de convivialidad diferentes, asimétricos y desiguales. ${ }^{2}$ Esos colectivos extensionistas negocian diferencias, asimetrías y desigualdades en procesos complejos de interacción cotidiana en cárceles, barrios, hospitales, escuelas, sindicatos, movimientos sociales, clubes barriales, para posibilitar y crear las condiciones del encuentro y el hacer junto a otros; y en ese mismo movimiento producen un conocimiento acerca del funcionamiento real de nuestras instituciones y espacios sociales que debe ser valorado. Adaptan y utilizan técnicas participativas, desde las heredadas de la educación popular o la investigación acción participativa, pasando por diferentes perspectivas etnográficas y el uso de herramientas metodológicas clásicas de la disciplina; incorporan enfoques teóricos que ponen el acento en las relaciones y las capacidades de agencia de los sujetos; diseñan modelos de investigación colectiva y dispositivos educativos alternativos; se enmarcan en la epistemología feminista; generan tecnologías de producción de datos acordes a los problemas sociales de la población que, a la vez, son productores y movilizadores de problemas públicos; integran saberes sociales y populares; estimulan agendas temáticas en diversos espacios 
sociales; acompañan procesos de formación militante y movilización popular; facilitan la comunicación entre los saberes disciplinares y las prácticas sociales habituales; capilarizan políticas públicas e identifican sus problemas efectivos de enfoque o implementación, etc. Los colectivos extensionistas son las radículas que enraízan a la universidad en el territorio a partir de su capacidad de producir enlaces con las tramas sociales que lo constituyen. Representan el diálogo de saberes y las ecologías del conocimiento en tiempo presente, no las declamadas, no las "retorizadas", sino las necesarias, las adecuadas al abordaje de los desafíos que presenta lo real en su forma mínima y molecular, pero fuertemente estructurante de nuestra condición social contemporánea.

\section{Dimensionando LA EXTENSIÓn EN LA FAHCE}

Sólo para dimensionar un poco, hagamos algunos números de la extensión universitaria en la Facultad de Humanidades y Ciencias de la Educación. Comparando el período 2008-2019 podremos ver en el gráfico siguiente como crecieron la cantidad de proyectos ejecutados, llegando a su pico en el ao 2017 a partir del cual se ingresó en una fase de descenso aunque con un piso que cuadruplica el punto de partida. Los equipos que forman parte de estos proyectos reflejan la diversidad disciplinar que coexiste en nuestra unidad académica y en la universidad.

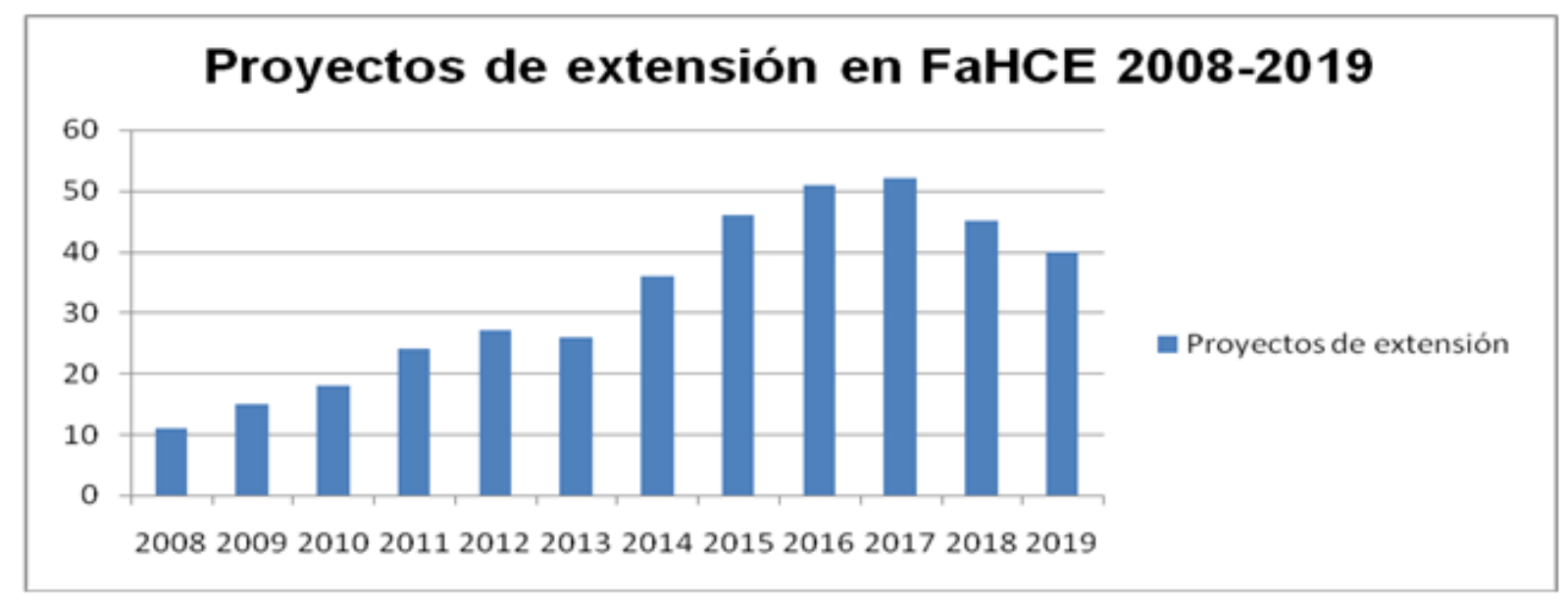

Fuente: Evaluación Institucional UNLP. Informe de Gestión de la Secretaría de Extensión FaHCE 2008-2019.

Por su parte las fuentes de financiamiento pueden ser un buen indicador no sólo de las fluctuaciones sino también de las compensaciones y el entramado de instituciones que dentro del sistema universitario sostienen las actividades de extensión. El progresivo compromiso de nuestra unidad académica en el sostenimiento financiero de las actividades de extensión es un dato destacable. 


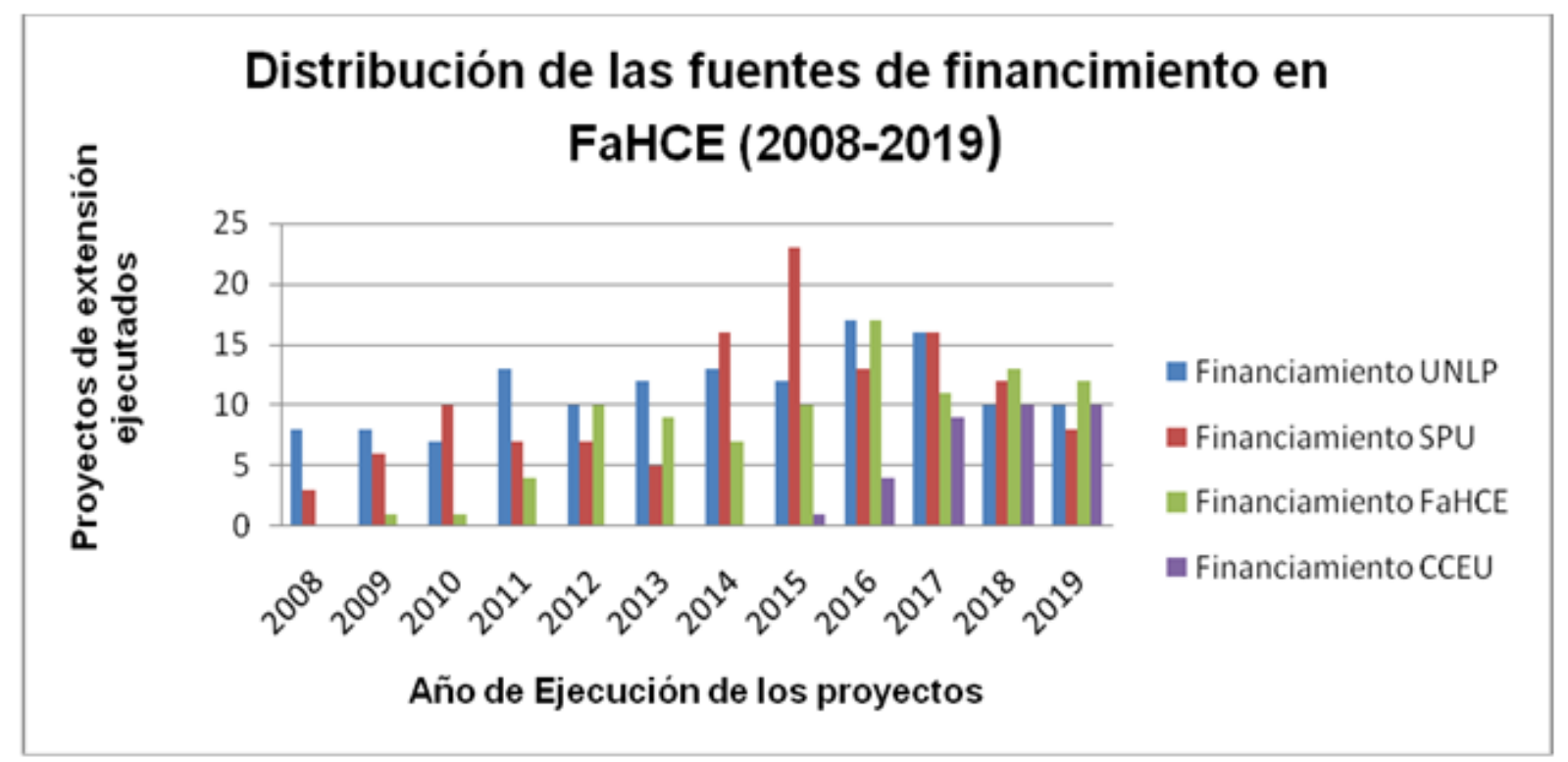

Fuente: Evaluación Institucional UNLP. Informe de Gestión de la Secretaría de Extensión FaHCE 2008-2019.

Un mapa sobre la distribución de las sedes de los proyectos durante el año 2018 también nos ayuda a visualizar la inserción territorial de las actividades de la Facultad más allá de sus muros. Puede observarse que la incidencia territorial de los proyectos concentra sus fuerzas en la región más inmediata (La Plata, Ensenada y Berisso), acompañando el proceso de regionalización de la UNLP en general. Al mismo tiempo, se puede señalar cierta vocación de unir los sitios de la periferia con las zonas centrales y también, mirando con atención las localizaciones en el mapa, podríamos deducir los circuitos y circulaciones que llevan a los equipos desde las áreas centrales de la ciudad hacia los márgenes y las periferias. La mayoría de los puntos marcados en el mapa representan las sedes de movimientos sociales, organizaciones barriales, instituciones educativas, clubes, centros comunitarios, locales sindicales, cooperativas, etc. 


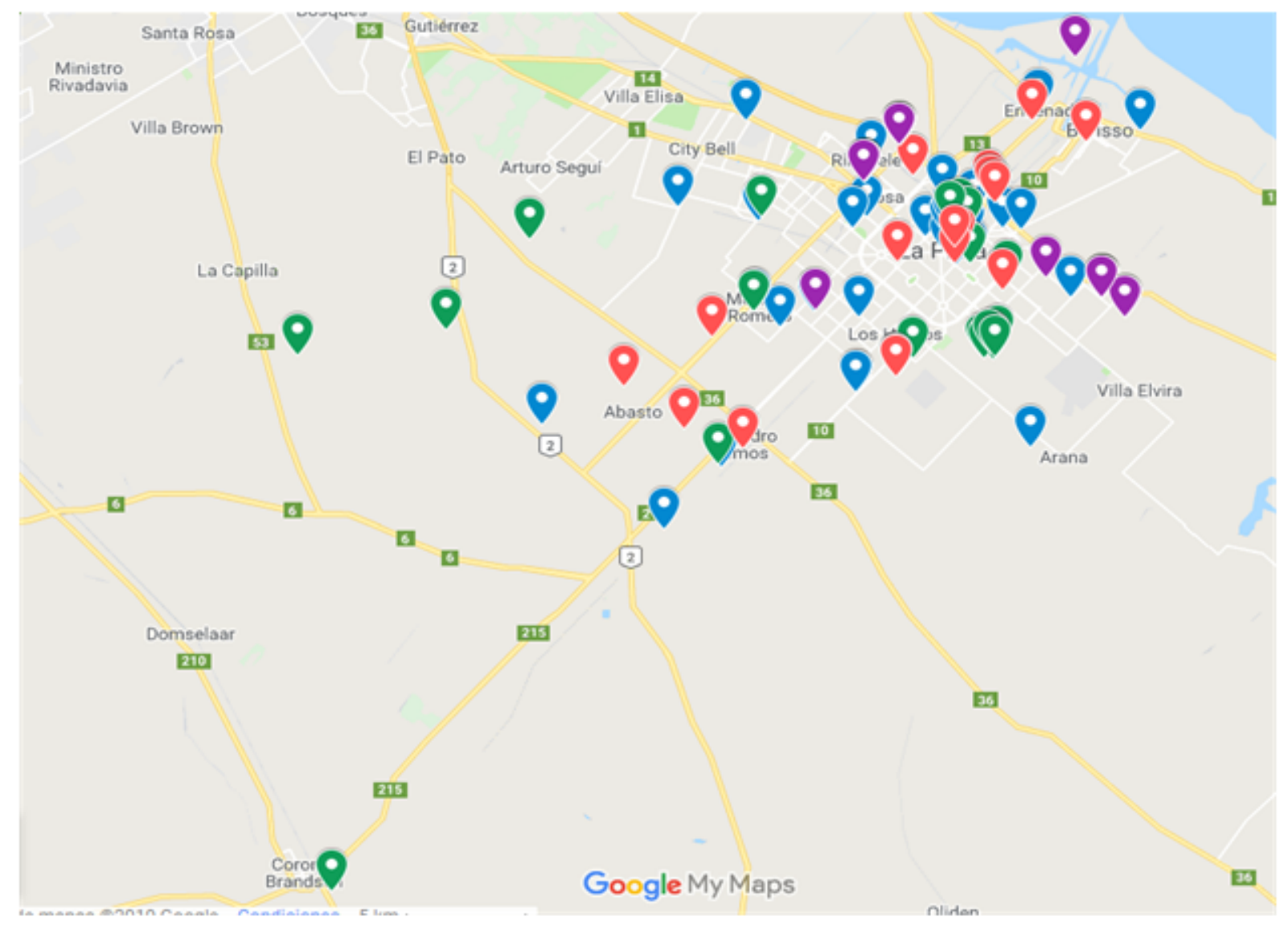

Fuente: Evaluación Institucional UNLP. Informe de Gestión de la Secretaría de Extensión FaHCE 2008-2019.

Por último, mencionemos dos experiencias que ponen a la extensión de la FaHCE en un período de mayor duración y consolidación. Una de ellas es el Programa de Educación Permanente para Adultos Mayores que cuenta con más de mil alumnos que participan en sus talleres y lleva veinticinco años de existencia. La otra experiencia tiene su origen en el departamento de sociología. El Centro Comunitario de Extensión Universitaria “La Unión y El Mercadito" resultó de una experiencia pionera, cuyas características y persistencia permiten dimensionar la duración y la consolidación de las actividades de extensión universitaria y su articulación con una perspectiva integral que vincula docencia, investigación y extensión. Concebido originalmente como proyecto de extensión en el seno del taller de investigación de cien horas "Pobreza: discusiones teórico-metodológicas" coordinado por Amalia Eguia y Susana Ortale, con el tiempo dio paso a la formación de un centro comunitario de extensión universitaria donde se articulan y cogestionan las actividades de la Universidad focalizada en los mencionados territorios. En este marco de trabajo se han formado varias generaciones de sociólogos y sociólogas en el despliegue de relevamientos integrales, articulaciones interinstitucionales, talleres de promoción de derechos, investigaciones multidimensionales sobre pobreza, trabajo, niñez, juventud, género, salud, alimentación, etc., y se continúan incorporando nuevas generaciones de estudiantes, graduadas y graduados.

\section{INVOLUCRAMIENTOS E IMAGINACIÓN SOCIOLÓGICA}

El enfoque sobre los modos de involucramiento nos conduce por una de las vías de problematización de las relaciones entre conocimiento y acción social. Las maneras en que las personas se involucran en el mundo nos permiten captar sus capacidades de actuar y sus maneras de experimentar el mundo en relación con sus intervenciones. El involucramiento es cambiante y convulsionado, y afecta la percepción 
de ese mundo y de las personas involucradas (Thévenot, 2016, p. 43). La cualidad humana de captar "lo que está ocurriendo en el mundo y comprender lo que está pasando con nosotros mismos como puntos diminutos de las intersecciones de nuestra biografía y de la historia dentro de la sociedad" es lo que Wright Mills llamaba imaginación sociológica (1994, p. 27). La imaginación sociológica varía en función de esos modos de involucramiento. Solo mediante una serie determinada de involucramientos se puede acceder a determinadas formas de imaginación sociológica. Si tenemos en cuenta la condición peculiar en la cual las inquietudes y preguntas acerca del funcionamiento de las relaciones sociales no pueden recurrir a un acervo de conocimiento concentrado ni disponible, ni a una idea de comprensión común que hace las veces de una mente colectiva a la que habría que alcanzar mediante algún tipo de técnica metodológica, sino que las respuestas a esas preguntas se hallan dispersas en una red específica de saberes, actores y acciones y que ninguna entidad dispone de ese saber representado de modo unitario, las formas de producción de conocimiento sobre lo social que se generan en las experiencias de extensión universitaria adoptan figura de redes de conocimiento o inteligencia distribuida. Se trata de situaciones de producción del conocimiento donde no hay ninguna persona, natural y/o artificial, que sea capaz de representar el conjunto del proceso (Hutchins, 1991, p. 36), sino que el conocimiento se produce en la colaboración práctica y cognitiva entre distintos actores que ocupan diferentes lugares dentro de esa red, y es la conexión intencionada de ese diferencial de posicionamientos desiguales el que habilita las circulaciones de saberes y conocimientos.

Lo que nosotros intentamos poner en juego fue un proceso dialógico para la construcción de conocimiento. Pero no era un diálogo diádico entre individuos abstractos y liberales, sino un diálogo complejo en el que había que partir de reconocer las diferencias fundadas en la desigualdad social para poder avanzar en la complejidad de la construcción de conocimiento que estábamos realizando. (Manzano, 2019, p. 80)

Para mostrar las conexiones entre los modos de involucramiento y la imaginación sociológica en la extensión universitaria tomemos cuatro sistematizaciones de experiencias (Jara Holliday, 2018) donde participaron estudiantes, docentes y graduados/as de nuestro departamento.

Después de cuatro años de trabajo fortaleciendo las redes de contra la violencia de género, las integrantes del proyecto de extensión "Nuevos nodos de Affidamento y prácticas en red. Promotoras comunitarias y estrategias situadas frente a la violencia de género" señalan las limitaciones de la denominada "ruta crítica" que debe recorrer una mujer cuando sufre violencia de género y sostienen la importancia de construir una noción de "ruta crítica ampliada" que integre las diferentes situaciones que se experimentan, dimensiones que implican y soportes sociales que hay que construir para que una mujer pueda superar las circunstancias a las que la somete la violencia patriarcal. Basadas en una epistemología feminista, destacan los relatos de las mujeres de sectores populares como la fuente de definición de la problemática y punto de partida de su abordaje:

La problemática emergía como acuciante en el seno del movimiento social, conformado en su mayoría por mujeres que viven en barrios populares de La Plata quienes relataban experiencias, en las cuales no sólo se identificaban situaciones vinculadas a la violencia de género sino también a las revictimizaciones que implicaba acudir a las instituciones del Estado. (Soza Rossi, P.; Trotta, L.; Rodríguez Durán, A.; Duarte, Y.; Giordano, A.; Muro, M.; Lofeudo, G., 2019).

Por su parte, luego de varios años de trabajo con personas migrantes de países limítrofes, los integrantes del proyecto "Identidad. La diferencia entre tener un derecho y poder ejercerlo"sostienen que el principal obstáculo para al acceso a las residencias legales no está en un trámite en particular, sino en la posibilidad de recorrer con éxito los "circuitos de identificación" por los que durante años circulan las personas migrantes, los papeles y las ventanillas del Estado, tratando de alcanzar su meta de ser "legales" en el país de residencia.

Este manual es un instrumento que resume parte de los conocimientos que adquirimos en el trabajo con colectivos y organizaciones de sectores populares. Lo escribimos con el objetivo de aportar una herramienta para facilitar el acceso a la documentación y potenciar las luchas por la ampliación de derechos. (...) buscamos visibilizar los problemas existentes en el acceso a documentos de identidad y residencias en su carácter social, situándolos en la agenda de lucha de las organizaciones 
populares (...) dimos prioridad a generar conocimiento sobre los problemas concretos que sufren los colectivos de migrantes, pueblos originarios y otros grupos vulnerados (...) las diferencias de acceso a los documentos de identidad hacen visibles las huellas de la desigualdad (...) [aquí] recuperamos el punto de vista (y los saberes) de las personas que necesitan acceder a estos documentos y recorren un circuito conformado por diversas agencias del Estado plagado de obstáculos... (Oyhandy, Pinedo y D’amico, 2014, pp. 19-25)

Al cumplir sus diez años de existencia, el colectivo Atrapamuros recoge su experiencia de trabajo en cárceles en el marco del proyecto "Educación popular en cárceles. El derecho social a la educación”y señalan la importancia de re-definir la perspectiva de género y el feminismo popular teniendo en cuenta los contextos estructurales y las trayectorias específicas de quienes habitan esos espacios.

Esta cartilla es un ejercicio de sistematización acerca de maneras posibles de encarar el trabajo en torno al género, y desde la perspectiva del feminismo popular, en un territorio específico: las cárceles de la Provincia de Buenos Aires. A partir del recorrido del trabajo extensionista y militante de 10 años, podemos señalar que se necesitan maneras específicas, contextuales y situadas para trabajar el género en estos contextos, cuestión que deviene de la especificidad estructural y subjetiva de esos espacios y de las trayectorias de quienes los habitan (Atrapamuros, 2019, p. 2).

Por su parte, los integrantes del proyecto "Alfabetización, educación y derechos" destacan como propio y significativo del trabajo extensionista lo colectivo, interdisciplinario y la capacidad de articular y adaptarse a los contextos para coproducir saberes con los movimientos territoriales.

...es un ámbito de trabajo realmente colectivo e interdisciplinario, como pocas veces puede lograrse en espacios de investigación o docencia, que nos ha llevado a cuestionar nuestros lugares comunes, nuestros enfoques disciplinarios y nuestras capacidades de comprensión, en función de un diálogo permanente con otros: alumnos o profesores de otras disciplinas, personas desocupadas o en situaciones de pobreza, miembros de una organización militante, graduados que no trabajan en la universidad (...) la organización con la que articulamos nuestros talleres es territorial, y con esto no solo queremos decir barrial, sino que nos referimos a un colectivo que se construye y se reproduce a partir de la apropiación de distintos espacios: una cooperativa, un comedor, un piquete, una movilización, un saber. En todos esos ámbitos, la construcción del "nosotros" en cuanto identidad colectiva no puede comprenderse sin su plataforma territorial. Nuestra interacción supuso entonces plegarnos desde nuestra práctica extensionista a dicha dinámica territorial y estar atentos y dispuestos a colaborar en las herramientas de apropiación de dichos espacios: ante la inundación, un acampe, un accidente laboral, etc. Todos esos sucesos, eventos y situaciones nos obligaron a revisar permanentemente nuestros saberes, nuestro rol de educadores y nuestra articulación con la organización (...) la propia dinámica suponía adaptarnos a contextos espaciales diversos y totalmente diferentes al áulico tradicional. (Torres F., Lafit F., Beliera A., Corsiglia Mura L., Martínez Ramírez F., Andolfo M. y Uehara L., 2018, pp. 12-17)

\section{UNA CIENCIA MODESTA}

¿Cómo entender las características de las contribuciones al conocimiento sobre lo social que arrojan estas experiencias de extensión universitaria? Si la sociología es un oficio, entonces el sociólogo puede considerarse un oficiante, cuya contribución medular, y al mismo tiempo modesta, consiste en identificar y estimular una capacidad distribuida en los más diversos órdenes de la existencia: la actitud humana de situarse socialmente y modelar sus relaciones y actuaciones, incluso en las condiciones de constricción estructural más estrechas.

Si tuviéramos que describir las características de este conocimiento podríamos decir que se trata de conocimientos que se producen en contextos dinámicos de utilización, no desde la lógica propia de la disciplina sino desde problemas en torno a la solución de situaciones concretas en los que se usan herramientas de las disciplinas. No se trata tanto de aplicar como de producir un conocimiento demandado que busca ser útil o adecuado para alguien o para algo (Dagnino, 2015). Requiere del trabajo colaborativo entre especialistas y no especialistas, de la construcción de consensos específicos entre actores heterogéneos en torno a presupuestos conceptuales, prácticas metodológicas y sus implicaciones extra-cognitivas (Torres Carrillo, 2015). La articulación de teorías, métodos y procedimientos se despliegan en función de la especificidad de los problemas a resolver y exige trabajo en equipo, diálogo y sensibilidad al contexto. La comunicación de 
los resultados está dirigida más a las poblaciones o grupos sociales que a las comunidades disciplinares, y por ello suele adoptar la forma de cartillas, manuales, folletos, informes, videos, antes que artículos o capítulos de libros (Rappaport, 2007). Los retos para este modo de producción del conocimiento no provienen tanto del balance crítico del saber acumulado por una disciplina sino de problemas social y políticamente desafiantes. Los lugares donde se localiza la producción de este conocimiento es plural, se puede generar más allá de los ámbitos académicos y exige flexibilidad en sus modos de organización y trabajo: grupos menos institucionalizados, equipos y redes temporales, roles y distribución de responsabilidades menos rígidas. Es un saber o un conjunto de saberes que razonan a partir de casos (Becker, 2016). Y como sostiene Alfonso Torres Carrillo (2015) no se avergüenza de ser una ciencia modesta, puede utilizar estrategias y técnicas sencillas y flexibles, y valora los lugares más diversos como ámbitos de producción de conocimiento.

Al involucrarse y configurar estilos o modos de hacer extensionista, los sociólogos y sociólogas, procrean y recrean las estructuras del conocimiento universitario, y al mismo tiempo, definen sus trayectorias formativas y profesionales. Se trata de un oficio de sociólogos y sociólogas donde la modestia y antiteoricismo estimula una apertura de los modos de producción de conocimiento que se dan en la escena universitaria actual. Entre todos estos elementos resulta interesante dejar fijado el esfuerzo singular de estos oficiantes: el intento sostenido de transformar las experiencias constituidas en sus involucramientos, a partir de la utilización de distintas herramientas técnicas, metodológicas y conceptuales provenientes de la disciplina en un sentido amplio, en resultados cognitivos que puedan desafiar un conocimiento establecido o agregar un nuevo conocimiento acerca de las tramas de relaciones sociales donde juegan sus apuestas.

\section{BiBLIOGRAFÍA}

Atrapamuros (2019). Hasta que todxs seamos libres. Construyendo feminismo popular en las cárceles. La Plata, Argentina.

Balerdi, S. (2018). Construyendo el reclamo. Etnografía de un conflicto por hábitat en La Plata, 2013-2017 (Tesis para optar el grado de Doctorado en Ciencias Sociales). Universidad Nacional de La Plata, La Plata.

Becker, H. (2016). Mozart, el asesinato y los limites del sentido común: cómo construir teoría a partir de casos. Buenos Aires: Siglo XXI.

Becker, H. (2015). Para hablar de la sociedad: la sociología no basta. Buenos Aires: Siglo XXI.

Castiñeiras, J. R. (1940). Historia de la Universidad Nacional de La Plata.La Plata: EDULP.

de Souza Santos, B. (2006). La sociología de las ausencias y la sociología de las emergencias: para una ecología de saberes. En Renovar la teoría crítica y reinventar la emancipación social (encuentros en Buenos Aires). Buenos Aires: CLACSO.

Dagnino, R. (2015). La Universidad latinoamericana del futuro que su sociedad está construyendo. Cuestiones de Sociología, 12. Recuperado de: http://www.cuestionessociologia.fahce.unlp.edu.ar

Femenías, M. L. y Soza Rossi, P. (2011). Para una mirada de género situada al sur. En M. L. Femenías y P. Soza Rossi (compiladoras), Saberes situados/Teorias transhumantes. La Plata: FaHCE-UNLP.

Gramsci, A. (2009). Los intelectuales y la organización de la cultura. Buenos Aires: Nueva Visión.

Jara Holliday, O. (2018). La sistematización de experiencias. Práctica y teorías para otros mundos posibles. Bogotá: CINDE.

Hutchins, E. (1991). Organizing Work by Adaption. Organization Science. 2(1), 14-39. The Institut of Managment Sciences.

Manzano, V. (2019). La producción de la circularidad del conocimiento. En F. Brugaletta, M. González Canosa, M. Starcenbaum y N. Welschinger, N. (Eds.), La politica cientifica en disputa: Diagnósticos y propuestas frente a su reorientación regresiva (2019). La Plata: Universidad Nacional de La Plata. Facultad de Humanidades y Ciencias de la Educación; CABA: CLACSO. (Andamios, 5. Serie perspectivas). Recuperado de: https://libros.fahce.un lp.edu.ar/index.php/libros/catalog/book/1 
Maria Sibylla Merian International Centre for Advanced Studies in the Humanities and social Social Sciences Conviviality-Inequality in Latin America (2017), "Conviviality in Unequal Societies: Perspectives from Latin America. Thematic Scope and Research Programme”, Mecila Working Paper Series, N. ${ }^{\circ}$ 1, Sao Paulo: Maria Sibylla Merian International Centre for Advanced Studies in the Humanities and Social Sciences ConvivialityInequality in Latin America.

Wright Mills, C. (1994) [1959]. La imaginación sociológica. México: FCE.

Oyhandy, A., Pinedo, J. y D'Amico, V. (Coords.). (2014). Identidad. La diferencia entre tener un derecho y poder ejercerlo : Un manual para facilitar el acceso a la documentación. La Plata: Universidad Nacional de La Plata. Facultad de Humanidades y Ciencias de la Educación. (Andamios, 1 Serie materiales). Recuperado de http://w ww.libros.fahce.unlp.edu.ar/index.php/libros/catalog/book/100

Pinedo, J. y Díaz, C. (2018). Políticas y prácticas de la extensión universitaria la luz de la democratización de la educación superior. En Crisorio R., Seré Quintero S. y Rocha Bidegain L. (coordinadores), Democratización de la educación superior. Politicas, actores e instituciones. Buenos Aires: Biblos.

Pinedo, J. (2019). La extensión universitaria como producción de conocimiento. Modalidades de articulación extensión-docencia-investigación. En F. Brugaletta, M. González Canosa, M. Starcenbaum y N. Welschinger (Eds.), La política cientifica en disputa: Diagnósticos y propuestas frente a su reorientación regresiva (2019). La Plata: Universidad Nacional de La Plata. Facultad de Humanidades y Ciencias de la Educación; CABA: CLACSO. (Andamios, 5. Serie perspectivas). Recuperado de: https://libros.fahce.unlp.edu.ar/index.php/libros/catalog/b ook/1

Rappaport, J. (2007). Más allá de la escritura: la epistemología de la etnografía en colaboración. Revista Colombiana de Antropología, 43, 197-229.

Pérez Rasseti, C. (2015). Tensionar la autonomía desde la extensión universitaria. Cuestiones de Sociología, 12. Recuperado de http://www.cuestionessociologia.fahce.unlp.edu.ar

Soza Rossi, P., Trotta, L., Rodriguez Durán, A., Duarte, Y., Giordano, A., Muro, M. y Lofeudo, G. (2019). Construyendo affidamento contra la violencia de género: de un nos-otras a nosotras. En prensa.

Thévenot, L. (2016), La acción plural: una introducción a la sociología pragmática, Buenos Aires: Siglo XXI.

Tomassino, H. y A. Cano (2016). Modelos de extensión universitaria en las universidades latinoamericanas en el siglo XXI: tendencias y controversias. Universidades, 67, 7-24. Unión de Universidades de América Latina y el Caribe, Distrito Federal, Organismo Internacional.

Torres, F., Lafit, F., Beliera, A., Corsiglia Mura, L., Martínez Ramírez, F., Andolfo M. y Uehara, L. (2018). La conquista $y$ defensa de nuestros derechos. La Plata: Universidad Nacional de La Plata. Facultad de Humanidades y Ciencias de la Educación. (Andamios, 3. Serie experiencias). Recuperado de https://libros.fahce.unlp.edu.ar/index.php/ libros/catalog/book/113

Torres Carrillo, A. (2015). Investigación acción participativa: entre las ciencias sociales y la educación popular. $L a$ piragua. Revista latinoamericana y caribeña de educación y politica, 41.

\section{Notas}

1 Para una discusión y propuesta acerca de la utilidad de las ciencias sociales y la ciencia pública en la Argentina se puede consultar el artículo de Revista Anfibia “¿Por qué hay que invertir en ciencias (también) sociales?”, que publicamos con Nicolás Welschinger y Victoria D'Amico: http://revistaanfibia.com/cronica/invertir-en-ciencias-sociales/?fbclid=Iw AR1tVzohgykdw1GTHnI8109J1Zn7BeJei4shUnWtDz-HCRFxmtzDflcv0xw

2 Conviviality: concepto analítico que circunscribe los modos de vivir juntos en contextos caracterizados por las asimetrías, las diferencias y las desigualdades. Admite gradaciones, de formas más horizontales a formas más verticales de convivencia. Intenta mostrar cómo las interacciones cotidianas constituyen contextos de negociación y resignificación de las posiciones sociales y las identidades culturales. Puede examinarse en tres dimensiones. Estructural (modelos o formas de convivencia): hace referencia a los macro contextos, las estructuras sociales, políticas, legales y los marcos institucionales, los espacios y las infraestructuras en los que ocurre la interacción, y tienen efectos (constrictivos, condicionantes, habilitantes) sobre los modos de convivencia. Negociaciones (articulaciones de la convivencia), procesos 
de disputa, negociación, consenso y regulación en diversas esferas de interacción en diferente nivel o escala (local, regional, nacional, internacional, global) y sus interrelaciones. Representaciones (imaginando la convivencia): cómo los actores sociales y los individuos imaginan y/o se representan la convivencia en sus respectivos espacios sociales. Incluye los aspectos discursivos y no discursivos, diferentes modos de conocimiento y re-conocimiento, creación y recreación de afectos y saberes producidos, circulados y transformados en y a través de los diferentes espacios de convivencia (Maria Sibylla Merian International Centre for Advanced Studies in the Humanities and social Social Sciences ConvivialityInequality in Latin America, 2017). 